\title{
Lithium deficient halo stars: abundance trends and enhanced rotation
}

\author{
Lisa M. Elliott ${ }^{1}$ and Sean G. Ryan ${ }^{2}$ \\ ${ }^{1}$ Centre for Stellar and Planetary Astrophysics, School of Mathematical Sciences, \\ Monash University, Clayton, Victoria, 3800 Australia \\ email: Lisa.Elliott@maths.monash.edu.au \\ ${ }^{2}$ Department of Physics and Adtronomy, The Open University, Walton Hall, Milton Keynes \\ MK7 6AA UK \\ email: s.g.ryan@open.ac.uk
}

\begin{abstract}
We present results from a high resolution study of six Li-depleted halo dwarfs. These stars exhibit Lithium abundances more than 0.5 dex below the Spite Plateau. The study is comprised of two parts: 1) a detailed abundance analysis covering many light, alpha, Fe-peak and neutron capture elements and 2) a study of rotational line broadening. We find that there are no common abundance anomalies, other than the Lithium depletion, that distinguish these stars from the Li-normal population. Four of the six stars however, show evidence of abnormally high projected rotation velocities. These findings support a non-standard depletion mechanism in Li-depleted halo stars, and emphasize their necessary exclusion from future studies of the Spite Plateau.
\end{abstract}

Keywords. Stars: abundances, stars: rotation

\section{Introduction}

While more than $90 \%$ of warm halo dwarfs show Lithium abundances close to the Spite Plateau (Spite \& Spite, 1982), a small fraction show extreme Lithium depletions. Often with undetectable Li $6707 \AA$ features, these stars are depleted by more than 0.5 dex below the plateau (eg Spite et al. (1984); Hobbs et al. (1991)). Previous abundance studies of the Lithium deficient halo stars (LDHS) have failed to identify any further common abundance anomalies (eg Norris et al. (1997)).

Ryan et al. (2002) found that three LDHS showed significant spectral line broadening and proposed that it was due to stellar rotation caused by mass transfer from an evolved donor. All three stars are spectroscopic binaries, providing extra evidence for such a scenario. Links have been made with field blue stragglers with Ryan et al. (2002) suggesting that the same mechanism may be responsible for both class of object.

This project extends the previous abundance and rotation studies to further invesitgate the chemical properties of LDHS and the link between Lithium deficiency and rapid rotation.

\section{Observations and Reductions}

The data covered a wavelength range between $3900 \AA$ and $6700 \AA$ and was collected on the $4 \mathrm{~m}$ WHT at La Palma, using the Utrecht Echelle Spectrograph $(\mathrm{R} \sim 45,000)$. Typical $\mathrm{S} / \mathrm{N}$ ratios obtained were between 50-100. Data reductions and EQW measurements used IRAF, and the abundances were calculated using WIDTH6 and KURUCZ model atmospheres. The rotation study involved spectrum synthesis using ATLAS9. Full details of the analysis can be found in Elliott et al. (2005, in prep) and Elliott \& Ryan (2005, in prep). 
Table 1. Abundance Trends and Rotation Characteristics

\begin{tabular}{lccccccc}
\hline Star & {$[\mathrm{Fe} / \mathrm{H}]$} & Teff & $\mathrm{A}(\mathrm{Li}) \dagger$ & Abundance Trends $\ddagger$ & Rotation & vsini $(\mathrm{km} / \mathrm{s})$ & Binary $\dagger$ \\
\hline HD97916 & -0.96 & 6375 & $<1.2$ & NORMAL & $\mathrm{Y}$ & 10.4 & $\mathrm{Y}$ \\
G202-65 & -1.36 & 6450 & $<1.67$ & NORMAL & $\mathrm{Y}$ & 8.6 & $\mathrm{Y}$ \\
G66-30 & -1.54 & 6440 & $<1.61$ & NORMAL & $\mathrm{Y}$ & 5.6 & $\mathrm{Y}$ \\
G139-8 & -2.27 & 6050 & $<1.39$ & Low Na, Sr \& Ba & $\mathrm{N}$ & $<1.9$ & $\mathrm{~N}$ \\
G122-69 & -2.67 & 5925 & $<1.10$ & NORMAL & $\mathrm{Y}$ & 4.7 & $\mathrm{~N}$ \\
G186-26 & -2.77 & 6260 & $<1.08$ & High Ba & $\mathrm{N}$ & $<2.5$ & $\mathrm{~N}$ \\
\hline
\end{tabular}

$\dagger A(L i)$ and binary status from Ryan et al. (2001) and references within.

$\ddagger$ Abundance trends with respect to the halo mean at the given $[\mathrm{Fe} / \mathrm{H}]$

\section{Results}

Table 1 shows a summary of results for the program stars. G139-8 and G186-26 show abnormal abundance characteristics when compared to the halo mean for stars of similar metallicity. It should be noted that although the abundances for these stars differ quite significantly from the halo mean, they are not outside the typical range for halo stars at low metallicity. For example, as noted in Norris et al. (1997), the high Ba abundance in G186-26 is also seen in $25 \%$ of Li-normal halo stars at that metallicity.

In terms of rotation, we identify a further LDHS, G122-69, with broadened lines. With an effective temperature of $5925 \mathrm{~K}$, this is by far the coolest rotating LDHS. The previously identified rotating stars all have effective temperatures above 6300K. For the other rotating objects we find good agreement with the measurements of Ryan et al. (2002) for G202-65 and G66-30 and Carney et al. (2001) for HD97916. We place upper limits on the remaining two stars, and these show rotation rates typical of Li-normal halo stars.

\section{Conclusions}

We have studied six LDHS and find that there are no abundance anomalies common to this group. We identify another LDHS showing rotationally broadened lines, bringing the total number to 5 (out of 8 studied). The four stars exhibiting the highest rotation rates are all believed to be in binary systems, giving further support to a mass-transfer scenario. Given that there is little evidence of rotation in the Li-normal population, it seems increasingly likely that there is a strong link between the rotation and the Lithium depletion. It also suggests that a non-standard depletion mechanism is responsible and implies that these stars should be ruled out of future studies of the Spite Plateau.

Lisa Elliott wishes to thank the IAU for partial funding to attend IAU Symposium 228, and the Australian Research Council for supporting this research.

\section{References}

Carney, B.W., Latham, D.W., Laird, J.B., Grant, C.E. \& Morse, J.A. 2001, AJ 122, 3419

Elliott, L.M. \& Ryan, S.G 2005, in prep.

Elliott, L.M., Ford, A., Ryan, S.G. \& Gregory, S. 2005, in prep.

Hobbs, L.M. \& Mathieu, R.D. 1991, PASP 103, 431

Kurucz, R.L. 1993, ATLAS9 stellar atmospheres programs

Norris, J.E., Ryan, S.G. \& Beers, T.C. 1997, ApJ 488, 350

Ryan, S.G., Beers, T.C., Kajino, T. \& Rosolankova, K. 2001, ApJ 547, 231

Ryan, S.G., Gregory, S.G., Kolb, U., Beers, T.C. \& Kajino, T. 2002, ApJ 571, 501

Spite, F. \& Spite, M. 1982, A\&GA 115, 357

Spite, M., Maillard, J.P. \& Spite, F. 1984, A\&A 141, 56 